\title{
Numerical simulations of unbounded cyclotron-maser emissions
}

\author{
D A VID C. SPEIRS, S. L. McCONVIL LE, \\ K. M. GILLESPIE, A. D. R. PHELPS \\ and K. RONALD \\ Physics Department, SUPA, University of Strathclyde, Glasgow, G4 0NG, UK \\ (david.c.speirs@strath.ac.uk)
}

(Received 28 August 2013; revised 28 August 2013; accepted 6 September 2013; first published online 12 November 2013)

\begin{abstract}
Numerical simulations have been conducted to study the spatial growth rate and emission topology of the cyclotron-maser instability responsible for stellar/planetary auroral magnetospheric radio emission and intense non-thermal radio emission in other astrophysical contexts. These simulations were carried out in an unconstrained geometry, so that the conditions existing within the source region of some natural electron cyclotron masers could be more closely modelled. The results have significant bearing on the radiation propagation and coupling characteristics within the source region of such non-thermal radio emissions.
\end{abstract}

\section{Introduction and background}

A variety of astrophysical radio emissions have been identified associated with non-uniform magnetic fields and accelerated particle streams (see Zarka 1992; Ergun et al. 2000; Treumann 2006). Numerous such sources, including planetary and stellar auroral radio emission, are spectrally well defined with a high degree of extraordinary (X-mode) polarisation. In particular, for the terrestrial auroral case it is now widely accepted (Bingham and Cairns 2000; Ergun et al. 2000; Bingham et al. 2004; Cairns et al. 2005) that such emissions are generated by an electron cyclotron-maser instability driven by a horseshoe-shaped electron velocity distribution. Such distributions are formed when particles descend into the increasing magnetic field of planetary/stellar auroral magnetospheres, where conservation of magnetic moment results in the conversion of axial momentum into rotational momentum forming an electron velocity distribution having a large spread in pitch factor. Theory has shown that such distributions are unstable to cyclotron emission in the X-mode. Inhomogeneous systems have also been examined (Cairns et al. 2008, 2011). Experiments and simulations carried out at the University of Strathclyde (Speirs et al. 2005, 2008, 2010; Gillespie et al. 2008; McConville et al. 2008; Ronald et al. 2008a,b, 2011) have investigated the electrodynamics of an electron beam subject to significant magnetic compression. Analytical theory (Bingham and Cairns 2000; Bingham et al. 2004; Cairns et al. 2005, 2008, 2011; Vorgul et al. 2011) developed at the University of St Andrews and at the STFC Rutherford Appleton Laboratory has greatly benefitted this experimental and numerical simulation research. More recently, particle-in-cell (PiC) simulations have been conducted to study the cyclotron-maser emission topology in the absence of radiation boundaries and as a function of various beam parameters, with the spatial growth of the instability and characteristics of radiation propagation documented.

\section{Results}

In the initial scaled laboratory experiments conducted at Strathclyde, an electron beam was injected from an annular explosive emission cathode and subjected to significant magnetic compression using a highly configurable solenoid arrangement. The beam transport characteristics and cyclotron-resonant beam-wave interaction within a circular cylindrical waveguide were simulated in $2.5 \mathrm{D}$ using the $\mathrm{PiC}$ code KARAT, with a typical electron beam trajectory presented in Fig. 1 for a magnetic mirror ratio of $B_{z} / B_{z 0}=34$ and peak magnetic flux density of $0.487 \mathrm{~T}$. There is a clear evidence of pitch angle expansion within the plotted trajectory, as indicated by the presence of a radially diffuse mirrored component within the convergent magnetic field region.

Within both the waveguide-bounded simulations and experiments, cyclotron resonant energy transfer was studied with near-cutoff Transverse Electric (TE) modes, which closely match the electromagnetic field polarization of the X-mode with respect to the magnetostatic field. Although radiation propagation and coupling was assumed to be in the forward-wave direction (with respect to the cathode), a significant number of cyclotronwave detunings resulted in backward-wave coupling. The magnetic field in the experiments and in the simulations was fine-tuned to allow the instability to vary between near perpendicular propagation to configurations where it could excite either slightly backward 


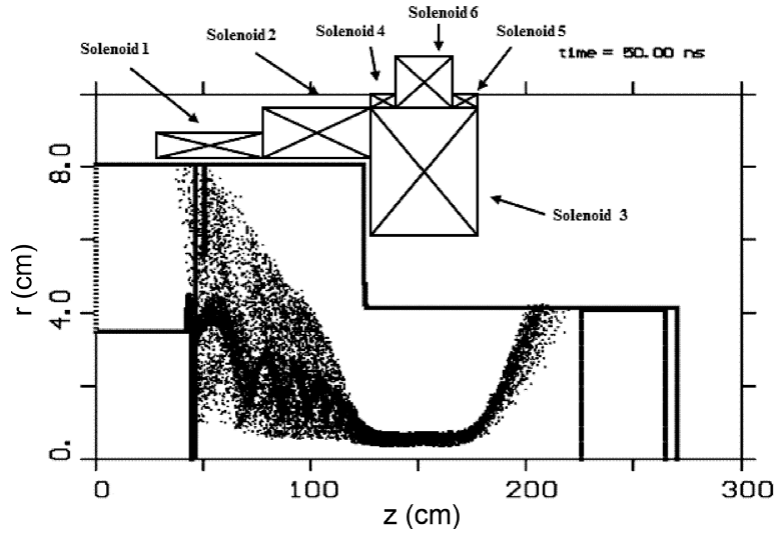

Figure 1. Experimentally consistent, waveguide-bounded PiC simulation geometry.

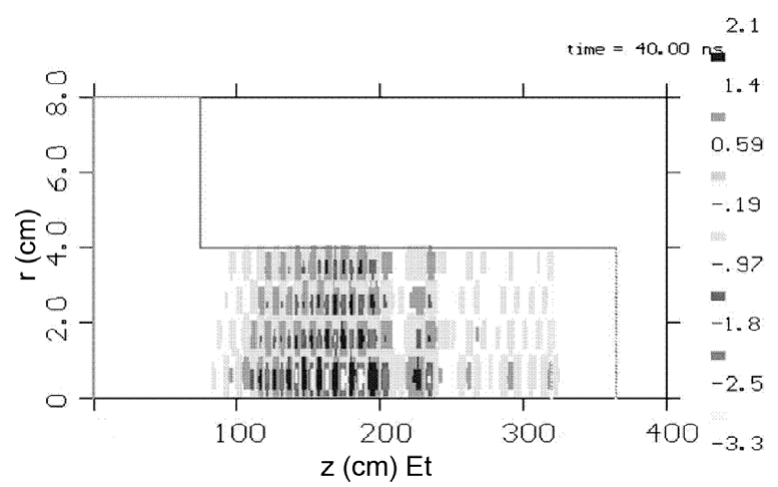

Figure 2. Contour plot of $E_{\theta}$ within a waveguide-bounded simulation geometry.

or forward propagating waves (at a slightly higher frequency). An example of the backward-wave regime is presented in Fig. 2, where a contour plot of $E_{\theta}$ from a $2.5 \mathrm{D}$ PiC simulation shows a more prominent backward-wave character to the cyclotron-wave coupling. A general feature of plasma micro-instabilities is that backward-wave coupling, provided several other conditions are satisfied, has the potential to lead to strong absolute instability growth (Allen and Phelps 1977) since there is an intrinsic feedback between the counter-streaming electrons and the propagating wave. In any specific electron beam/plasma configuration, however, a detailed analysis is required to establish whether an instability is absolute, or convective, in character.

In order to bridge between the waveguide-bounded experiments and an unbounded astrophysical scenario, we conducted $\mathrm{PiC}$ simulations to study the horseshoe cyclotron-maser instability in the absence of radiation boundaries. For the purpose of simulating the unbounded interaction geometry, a $44-\mathrm{cm}$ thick region of radially increasing conductivity was defined around the 4-m propagation path of a $20 \mathrm{keV}, 14 \mathrm{~A}$ electron beam. This represented an idealised absorber of electromagnetic radiation, inhibiting reflection and the formation of boundary resonant eigenmodes. Other simulation parameters include a predefined electron beam horseshoe

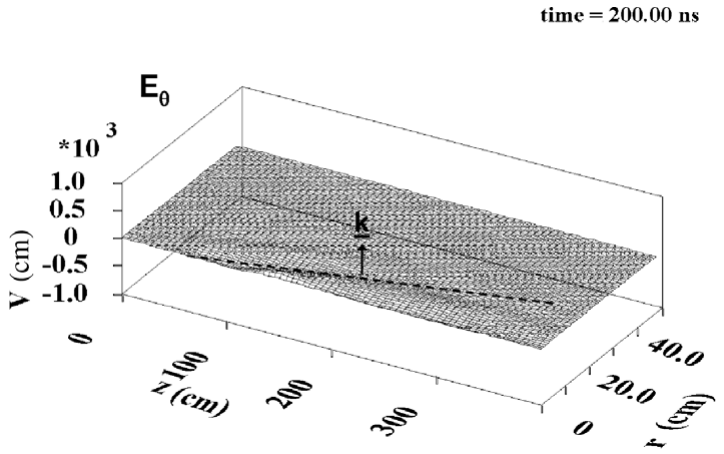

Figure 3. 3D contour plot of $E_{\theta}$ within an unbounded simulation geometry.

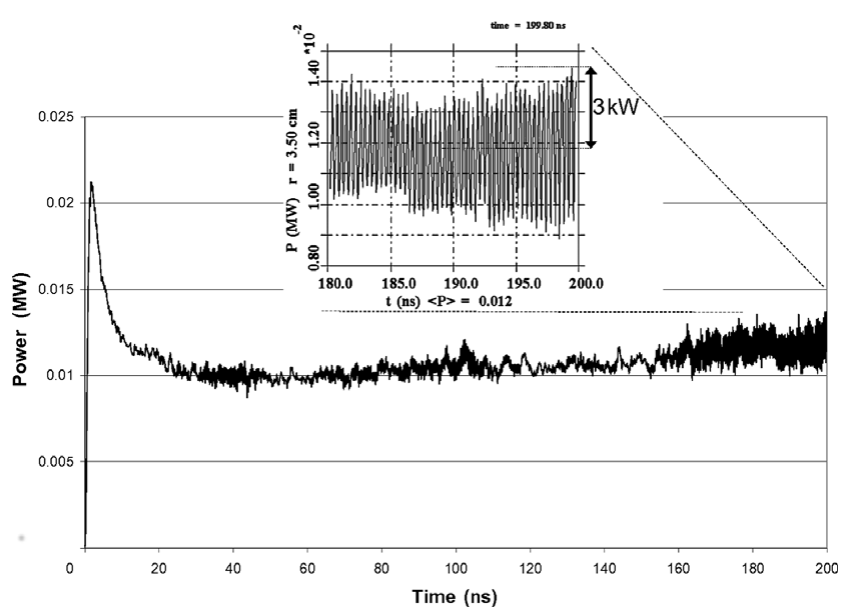

Figure 4. Radial Poynting flux temporal evolution, measured in a plane at $\mathrm{r}=3.5 \mathrm{~cm}$ and spanning the length of the simulation.

distribution, comprising a pitch spread of $\alpha=v_{\perp} / v_{z}=$ $0 \rightarrow 9.5$ and a uniform axial magnetic flux density of 0.1 $\mathrm{T}$. The simulations demonstrate a clear relaxation of the electron horseshoe distribution via the cyclotron-maser instability, with radio frequency (rf) output generated in the X-mode at the electron cyclotron frequency and with a small negative axial wavenumber (Fig. 3). This is evidenced by the oblique propagation angle of the wavefront highlighted in Fig. 3, which factoring in the differing spatial scales of $\mathrm{z}$ and $\mathrm{r}$ axes corresponds to a backward-wave propagation angle of few degrees.

A plot of the corresponding radial Poynting flux is presented in Fig. 4 measured in a plane at $\mathrm{r}=3.5 \mathrm{~cm}$. A DC offset is present in the measurement due to lowfrequency electromagnetic field components associated with the electron beam propagation. After $180 \mathrm{~ns}$, a peak rf output power of $3 \mathrm{~kW}$ was measured corresponding to an $\mathrm{rf}$ conversion efficiency of $1.1 \%$ - comparable with the estimate of $1 \%$ for the astrophysical phenomena (Gurnett 1974).

\section{Conclusions}

PiC simulations have been conducted to study the electrodynamics and emission topology of the cyclotronmaser instability responsible for auroral magnetospheric 
radio emission and the generation of non-thermal radio emission in other astrophysical contexts (Treumann 2006). Initial waveguide-bounded PiC simulations and laboratory experiments show for numerous near-cutoff resonance regimes, a tendency for cyclotronwave coupling to favour a backward-wave character. This trend is reflected in the results of unbounded PiC simulations of cyclotron-wave coupling, where radiation propagation has been observed with a distinctive backward-wave character to it, typically directed in a slightly backward direction, only a few degrees away from the perpendicular to the magnetostatic field. The corresponding rf conversion efficiencies are comparable with estimates for the astrophysical phenomena (Gurnett 1974; Treumann 2006; Bingham et al. 2013).

\section{Acknowledgements}

The authors thank R. A. Cairns, R. Bingham and I. Vorgul for many helpful discussions, V.P. Tarakanov for his expert advice on using the PiC code KARAT and the EPSRC for supporting this research. The authors would also like to acknowledge the encouragement, support and advice they received from the late Padma Shukla as a visiting professor at the University of Strathclyde.

\section{References}

Allen, J. E. and Phelps, A. D. R. 1977 Waves and microinstabilities in plasmas-linear effects. Rep. Prog. Phys. 40(11), 1305-1368.

Bingham, R. and Cairns, R. A. 2000 Generation of auroral kilometric radiation by electron horseshoe distributions. Phys. Plasmas 7(7), 3089-3092.

Bingham, R., Kellett, B. J., Cairns, R. A., Vorgul, I., Phelps, A. D. R., Ronald, K. and Speirs, D. 2004 Cyclotron maser radiation in space and laboratory plasmas. Contrib. Plasma Phys. 44(5-6), 382-387.

Bingham, R., Speirs, D. C., Kellett, B. J., Vorgul, I., McConville, S. L., Cairns, R. A., Cross, A. W., Phelps, A. D. R. and Ronald, K. 2013 Laboratory astrophysics: investigation of planetary and astrophysical maser emission. Space Sci. Rev. 10.1007/s11214-013-9963-z, 1-19.

Cairns, R. A., Speirs, D. C., Ronald, K., Vorgul, I., Kellett, B. J., Phelps, A. D. R. and Bingham, R. 2005 A cyclotron maser instability with application to space and laboratory plasmas. Phys. Scripta T116, 23-26.

Cairns, R. A., Vorgul, I. and Bingham, R. 2008 Cyclotron maser radiation from an inhomogeneous plasma. Phys. Rev. Lett. 101, 215003.

Cairns, R. A., Vorgul, I., Bingham, R., Ronald, K., Speirs, D. C., McConville, S. L., Gillespie, K. M., Bryson, R., Phelps, A. D. R., Kellett, B. J. et al. 2011 Cyclotron maser radiation from inhomogeneous plasmas. Phys. Plasmas 18, 022902.

Ergun, R. E., Carlson, C. W., McFadden, J. P., Delory, G. T., Strangeway, R. J. and Pritchett, P. L. 2000 Electroncyclotron maser driven by charged-particle acceleration from magnetic field-aligned electric fields. Astrophys. $J$. 538 $(1,1), 456-466$.

Gillespie, K. M., Speirs, D. C., Ronald, K., McConville, S. L., Phelps, A. D. R., Bingham, R., Cross, A. W., Robertson, C. W., Whyte, C. G., He, W. et al. 2008 3D PiC code simulations for a laboratory experimental investigation of auroral kilometric radiation mechanisms. Plasma Phys. Control. Fusion 50, 124038.

Gurnett, D. A. 1974 Earth as a radio-source - terrestrial kilometric radiation. J. Geophys. Res. 79(28), 42274238.

McConville, S. L., Speirs, D. C., Ronald, K., Phelps, A. D. R., Cross, A. W., Bingham, R., Robertson, C. W., Whyte, C. G., He, W., Gillespie, K. M. et al. 2008 Demonstration of auroral radio emission mechanisms by laboratory experiment. Plasma Phys. Control. Fusion 50, 074010.

Ronald, K., McConville, S. L., Speirs, D. C., Phelps, A. D. R., Robertson, C. W., Whyte, C. G., Gillespie, K. M., Cross, A. W. and Bingham, R. 2008a Electron beam measurements for a laboratory simulation of auroral kilometric radiation. Plasma Sources Sci. Technol. 17, 035011.

Ronald, K., Speirs, D. C., McConville, S. L., Gillespie, K. M., Phelps, A. D. R., Bingham, R., Vorgul, I., Cairns, R. A., Cross, A. W., Robertson, C. W. et al. 2011 Auroral magnetospheric cyclotron emission processes: numerical and experimental simulations. Plasma Phys. Control. Fusion 53, 074015.

Ronald, K., Speirs, D. C., McConville, S. L., Phelps, A. D. R., Robertson, C. W., Whyte, C. G., He, W., Gillespie, K. M., Cross, A. W. and Bingham, R. 2008b Radio frequency resonator structure and diagnostic measurements for a laboratory simulation of auroral kilometric radiation. Phys. Plasmas 15, 056503.

Speirs, D. C., McConville, S. L., Gillespie, K. M., Ronald, K., Phelps, A. D. R., Cross, A. W., Bingham, R., Robertson, C. W., Whyte, C. G., Vorgul, I. et al. 2008 Numerical simulation of auroral cyclotron maser processes. Plasm Phys. Control. Fusion 50, 074011.

Speirs, D. C., Ronald, K., McConville, S. L., Gillespie, K. M., Phelps, A. D. R., Cross, A. W., Bingham, R., Robertson, C. W., Whyte, C. G., He, W. et al. 2010 Numerical investigation of auroral cyclotron maser processes. Phys. Plasmas 17, 056501.

Speirs, D. C., Vorgul, I., Ronald, K., Bingham, R., Cairns, R. A., Phelps, A. D. R., Kellett, B. J., Cross, A. W., Whyte, C. G. and Robertson, C. 2005 A laboratory experiment to investigate auroral kilometric radiation emission mechanisms. J. Plasma Phys. 71(5), 665-674.

Treumann, R. A. 2006 The electron-cyclotron maser for astrophysical application. Astron. Astrophys. Rev. 13(4), 229-315.

Vorgul, I., Kellett, B. J., Cairns, R. A., Bingham, R., Ronald, K., Speirs, D. C., McConville, S. L., Gillespie, K. M. and Phelps, A. D. R. 2011 Cyclotron maser emission: Stars, planets, and laboratory. Phys. Plasmas 18, 056501.

Zarka, P. 1992 The auroral radio emissions from planetary magnetospheres - what do we know, what don't we know, what do we learn from them? In: Planetary Magnetospheric Physics II (ed. M. G. Kivelson, S. K. Runcorn, N. F. Ness and W. H. Ip). Amsterdam, The Netherlands: Elsevier; Adv. Space Res., 12(8), 99-115. 